\title{
From Therapeutic Relaxation to Mindfulness in the Twentieth Century
}

\author{
Ayesha Nathoo
}

\begin{abstract}
This chapter is based on a talk given by historian Ayesha Nathoo at the 'Mindfulness Unpacked' symposium at Wellcome Collection in February 2016, which was linked to the 'Tibet's Secret Temple' exhibition. While the exhibition contextualized mindfulness within the Buddhist tradition, Ayesha's research on the history of therapeutic relaxation provided an opportunity to demonstrate the social and structural links between secular mindfulness and twentieth-century relaxation practices.
\end{abstract}

Keywords Muscle relaxation - Mind-body medicine - Rest - Stress . Twentieth-century history of medicine

Today's Western 'mindfulness movement' is often portrayed as a sign of and remedy for our restless, overloaded times. Contemporary mindfulness-based interventions comprise treatments for pain, mental illness and stress management and have become an integral part of preventive medicine, lifestyle and well-being agendas. In Britain, the 2015 Mindfulness All-Party Parliamentary Group report 'Mindful NationUK', recommended that mindfulness be taught within schools, prisons, the workplace, and doctor and teacher training courses; there is now a burgeoning

\footnotetext{
A. Nathoo $(\bowtie)$

University of Exeter, Exeter, United Kingdom

e-mail: A.Nathoo@exeter.ac.uk

(C) The Author(s) 2016

F. Callard et al. (eds.), The Restless Compendium, DOI 10.1007/978-3-319-45264-7_9
} 
industry of self-help books, audio recordings, apps and group classes on offer. As its popularity has increased, criticisms are being voiced, particularly over the individualistic, commodifiable form of 'McMindfulness', seen as complicit with a neoliberal political agenda. How have we reached this point historically and socially? And is it really all so new?

The history of mindfulness tends to be presented in one of two ways: firstly, a history that reaches back to millennia-old Buddhist teachings, from which the meditative practices and ethical frameworks derive. And secondly, a narrative starting in 1979 when the molecular biologist Jon Kabat-Zinn established what became the mindfulness-based stress reduction (MBSR) programme at the University of Massachusetts Medical School, following a 'vision' he'd experienced during a vipassanã meditation retreat of adapting and applying Buddhist teachings to mainstream medical and popular settings. This chapter provides an alternative historical lens. It demonstrates how Western, secular, mindfulness-based interventions both structurally and culturally built upon teachings and understandings of therapeutic relaxation which were developed from the early decades of the twentieth century.

An oft-cited definition of mindfulness, outlined by Kabat-Zinn, is the awareness that comes from 'paying attention in a particular way: on purpose, in the present moment, and nonjudgementally'. Mindfulness practitioners often clearly demarcate relaxation from mindfulness, describing relaxation as a potential 'side-effect' of mindfulness, but not an aim in and of itself. Although relaxation may ensue, the intention is not to try to achieve any particular state, but rather to be open to whatever thoughts and feelings pass through the mind and body, moment by moment. My aim here is neither to reduce mindfulness to relaxation, nor to enter debates around precise definitions and comparative methodologies. Instead, I explore the ways in which the framing, proliferation and application of relaxation therapies helped to pave the way for today's form of mindfulness in the West and its widespread uptake. Nonetheless, it is relevant to note that KabatZinn's original course was called the 'Stress Reduction and Relaxation Program', and relaxation terminology was embedded in his early teachings and publications before being disavowed. ${ }^{2}$

Overlaps between mindfulness and relaxation have changed over time, as have scientific and cultural meanings of what it means to be both 'mindful' and 'relaxed'. In the interwar years, the Chicago physician and psychologist Edmund Jacobson advocated a new and narrower definition of the term relaxation, shifting away from vernacular connotations linked to recreational pursuits. Moreover, he distinguished 
between the commonly linked notions of 'relaxation' and 'rest', arguing that one could be resting but tense, or active yet at ease. Jacobson developed an intricate system of 'neuro-muscular relaxation', promoted as a physiologically based, scientifically valid technique. It was designed both to prevent and treat a wide array of physical and mental conditions and enhance experiences of everyday living, if comprehensively learnt and practised. Based on the premise that it is 'impossible to be tense and relaxed at the same time', and that thought itself produced muscular tension, his technique of 'progressive muscle relaxation' was a way to alleviate both physical and emotional tension. Jacobson's method involved systematically recognizing and releasing muscular tension, in order to quieten body and mind. He also advocated 'differential relaxation', whereby only muscles essential to a particular task were used and all others were relaxed, thereby eliminating unnecessary tension. Using an instrument that could detect and display minute changes in neuromuscular electrical activity, tension became something that could be observed, quantified and treated.

Jacobson's 1934 book, You Must Relax: A Practical Method of Reducing the Strains of Modern Living, outlined the myriad therapeutic applications of his system of relaxation: from nervous illness, to heart disease, insomnia and indigestion - all symptomatic of tense, overactive minds and bodies unable to keep up with the pace and demands of modern living. Jacobson considered relaxation to be far superior to the traditional remedy for many of these conditions - bed rest - given that one could remain tense when resting or even sleeping, and residual tension could serve to increase rather than decrease restlessness or 'nervousness'. Rather than a common, natural state of 'rest', muscular relaxation was a skill that needed to be taught, involving weekly instruction with a qualified physician, and one or two hours of home practice per day. Once mastered, short spells of deep relaxation could be used as a means of increasing efficiency and enjoying 'modern civilization without burning the candle at both ends' ${ }^{3 \mathrm{i}}$

Placing his method firmly in the Western, scientific, secular domain, Jacobson distanced his technique from relaxation practices and ideologies that developed in parallel, influenced by occultism and the hypnotic tradition - in particular, breathing and postural exercises taught by New Thought author Annie Payson Call, and 'autogenic training' using

\footnotetext{
${ }^{i}$ See Chap. 15.
} 
visualizations, formulated by the German psychiatrist Johannes Schultz. Despite these differences, and Jacobson's exclusive focus on bodily sensations, common to all of these techniques was a belief in the connection between mind and body and the therapeutic benefits of relaxation. They also required expert teaching and disciplined practice in order to cultivate a skill and an altered relationship to modernity. Much like mindfulness today, the aim of relaxation training was not to change industrialized socio-political structures and environments, but to enable individuals to relate differently to these contexts.

Muscular relaxation was enthusiastically taken up in Britain in the 1930s, especially within the performing arts and for speech therapy. Practitioners such as speech therapist M. A. Richardson and E. J. Boome from London County Council developed classes for stammering children, and disseminated the principles and ideology of relaxation therapy to wider audiences. Their 1938 publication, Relaxation in Everyday Life, described how habitually practising therapeutic or 'curative' relaxation might not only help with specific ailments but profoundly change an individual's entire outlook, and by osmosis impact on wider cultures of care. ${ }^{4}$ The authors stressed the importance and responsibility of parents, especially mothers, to learn to relax for their own health and well-being and in order to foster a better home environment for their children. ${ }^{\text {ii }}$ Teachers and healthcare workers too were identified as professionals for "whom the study of relaxation should be essential', in order to benefit the community at large. Over 80 years before the 'Mindful Nation UK' report, Boome and Richardson declared: 'We hope that the time will eventually come when [relaxation] will be considered an indispensable part of the training of those who are in contact with children and the sick - teachers, and nursery and hospital nurses'. ${ }^{5}$

Although relaxation training was applicable to all, it became especially popular amongst middle-class, child-bearing women in Britain, owing largely to the work of the obstetrician Grantly Dick-Read. In a pronatalist, eugenic socio-political context, concerned with the 'fitness' of the nation, Dick-Read sought to explain and address the relatively low birth rate among middle-class women. He suggested that their reproduction rates were being impeded by fear of the pain of childbirth. Relaxation was a means to break this fear-tension-pain cycle and enable a 'natural childbirth' (a term he coined in 1933), reduce the need for analgesics, and allow childbirth to become a positive, even spiritual experience. Although

ii See Chap. 10. 
sidelined by his medical colleagues, Dick-Read gained the support of influential midwives and physiotherapists who helped design, teach and implement relaxation exercises for antenatal women. Most significantly he developed a following amongst middle-class women themselves. ${ }^{6}$

In 1956, Prunella Briance - a mother who had endured a traumatic stillbirth of her child under conventional obstetric care - set up the still widely popular National Childbirth Trust (originally the Natural Childbirth Association), to promote the teachings of Dick-Read. Relaxation exercises in preparation for childbirth soon became a routinized part of antenatal care (Fig. 9.1). ${ }^{7}$ In the postwar decades, antenatal teachers became leading proponents of relaxation, quick to adapt and extend teachings from the antenatal to postnatal period, both creating and meeting a demand to help mothers better manage the multiple challenges of parenthood. Meanwhile, physiotherapists extended principles of relaxation for childbirth to relaxation for pain management more generally, establishing an enduring

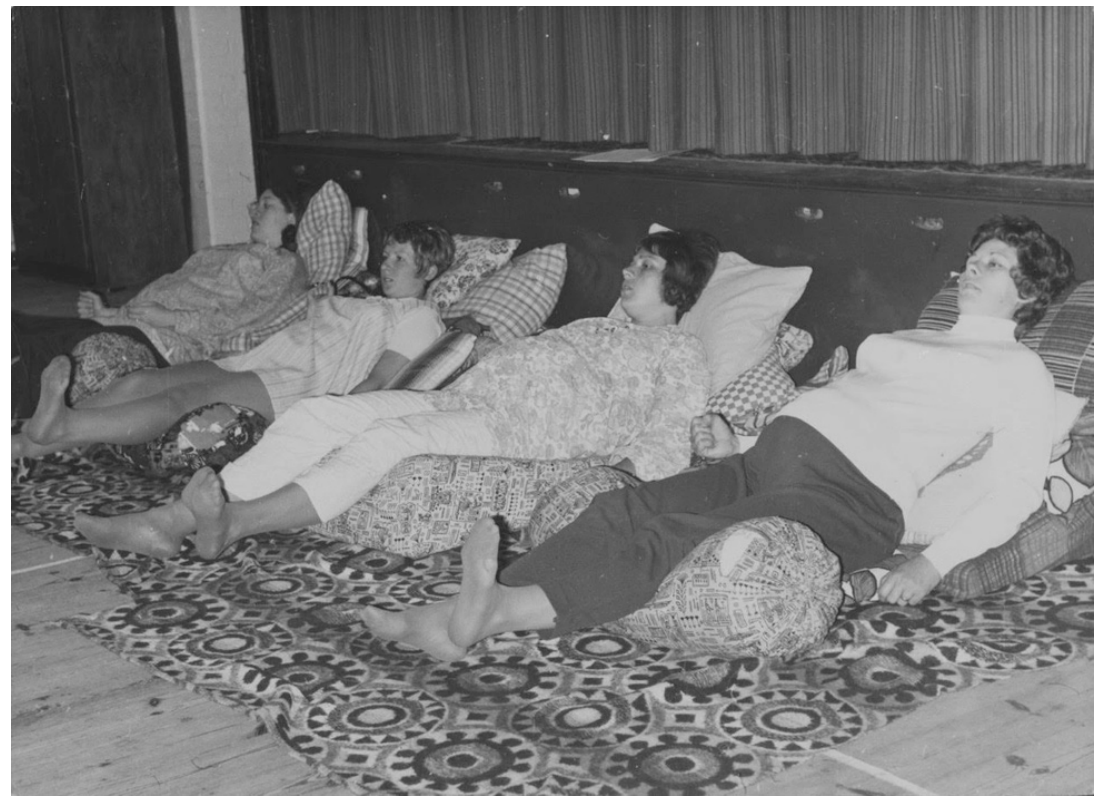

Fig. 9.1 NCT Havering Branch: antenatal class photograph. (Credit: NCT/ Wellcome Library, London, Wellcome Images, L0079526, released under a Creative Commons Attribution 4.0 International License (CC BY 4.0)) 
therapeutic role for these techniques. Relaxation became relevant for men too, especially ostensibly 'coronary-prone', overworked 'Type A' businessmen, seen as a particularly vulnerable group as physiological, psychological and epidemiological research into 'stress', 'risk-factors' and 'coping mechanisms' for heart disease developed in this period. ${ }^{8}$

By the 1970s group relaxation classes, as a means of 'stress management', were being offered and structured in much the same way as mindfulness classes are today. Courses involved weekly sessions guided by an 'expert' teacher, with audio recordings available for daily home practice. Course participants learnt breathing and 'deep relaxation' techniques, and were encouraged to adapt and apply 'differential' relaxation principles to everyday living. As well as relaxation instruction, classes incorporated health educational messages, group discussions, and basic scientific and physiological explanations of the body's 'fight or flight' response and the perceived detrimental impact on health and well-being of chronic exposure to stress. ${ }^{\text {ii }}$ Relaxation was promoted as relevant and helpful for the well-being of individuals, homes, the workplace and society at large. It was framed as a safe, effective alternative to drugs for anxiety, insomnia and hypertension, and a way not only to treat ill-health, or 'dis-ease', but to encourage healthy lifestyles and boost well-being.

National and local television and radio programmes in the 1970s regularly featured relaxation discussion and teaching; and health educators, physiotherapists, general practitioners, antenatal instructors, physical education teachers, psychologists and psychotherapists all had a stake in relaxation training and promotion, and contributed to the growing selfhelp literature. But alongside the enthusiasm came words of caution. As one doctor signalled in a medical journal review:

A single trip to the bookshop may well for many patients replace a series of repeat prescriptions for a benzodiazepine. Naturally however, the same caution applies to the use of relaxation as to the use of tranquillizers: one must be certain that one is not merely suppressing symptoms when it is more appropriate, if more difficult, to deal directly with an underlying cause. ${ }^{9}$

He also expressed concern that therapeutic relaxation instruction was aimed mainly at an 'upper-middle class audience' - the demographic who could most likely afford the training and the time to learn and effectively keep up practice. Relaxation practitioners, meanwhile, were concerned

iii Cf. Chap. 12. 
about upholding the quality of teachers and demarcating 'scientific' relaxation from numerous other unregulated 'alternative therapies'. All of these issues chime with those facing mindfulness today.

Although many Western practitioners distanced therapeutic relaxation from mystical traditions, others explicitly connected it to Eastern meditative practices. One relaxation advocate explained: 'A person who learns how to relax completely - or meditate, as the Eastern teachers call it acquires the capacity of freeing himself from fears and useless brooding over bad experiences'. ${ }^{10}$ Yoga scholar Mark Singleton has demonstrated how yoga was reconstituted as it proliferated in the West in the postwar decades, drawing more from contemporary, Western relaxation techniques and ideologies, than ancient Indian teachings. ${ }^{11}$ While the New Age narrative of 'Eastern wisdom' appealed to some, yoga and meditation reached far beyond the 1960s counterculture movement, and Christian as well as dharmic meditative traditions appropriated the therapeutic framework of relaxation. In the early 1970s, for example, Reverend Geoffrey Harding, head of the Churches Council for Health and Healing, founded the Relaxation Society and held lunchtime relaxation classes for city workers in his City of London church. 'By relaxing', he explained, 'we discover our own inner life, and begin to enjoy the "eternal life" that Christ was talking about. This means much more than "future life". It means Life, here and now, "Life within oneself" (St. John v. 26)'.12

The 1975 international bestseller, The Relaxation Response, by Harvard cardiologist Herbert Benson, ${ }^{13}$ further coupled relaxation with meditation, and significantly bolstered claims of the therapeutic potential of such techniques. The book extended Benson's prior research, alongside colleague Robert Keith Wallace, into the physiological effects of transcendental meditation (TM), to include a host of 'ancient' and 'modern' methods. These included progressive muscle relaxation, yoga, meditation, autogenic training as well as repetitive prayer, all of which, Benson argued, produced similar underlying physiological changes. He defined the relaxation response as 'a physical state of deep rest that changes the physical and emotional responses to stress .... and opposite of the fight or flight response'. Through lowering metabolism, blood pressure, heart rate and respiratory rate, amongst other physical indicators, Benson considered the relaxation response, however elicited, to be a powerful antidote to a whole array of stress-related conditions.

Benson significantly raised the profile of relaxation practices and 'mindbody medicine' in medical and popular arenas, no doubt helping to create 
a more receptive environment for Kabat-Zinn's introduction of mindfulness into clinical settings. As Kabat-Zinn recently reflected, 'naming is very important in how things are understood and either accepted or not'. Labelling the original course the 'Stress Reduction and Relaxation Program' in 1979 was therefore a strategic choice. 'Mindfulness meditation' clearly drew on long personal, cultural and historical encounters with Buddhist teachings, ${ }^{14}$ but Kabat-Zinn's clinical programme also significantly built on modern relaxation theories and practices as popularly accepted and therapeutically understood. As he recalled:

From the beginning of MBSR, I bent over backward to structure it and find ways to speak about it that avoided as much as possible the risk of it being seen as Buddhist, 'New Age', 'Eastern Mysticism' or just plain 'flakey'. To my mind this was a constant and serious risk that would have undermined our attempts to present it as commonsensical, evidence-based, and ordinary, and ultimately a legitimate element of mainstream medical care. ${ }^{15}$

In the late 1980s, the classes were reestablished in the Stress Reduction Clinic, 'emphasizing that it was a clinical service... in the Department of Medicine'. The programme was renamed 'mindfulness-based stress reduction' in the early 1990s, 'to differentiate the approach from many programmes that also used the term stress reduction or stress management but that had no dharma foundation whatsoever'. ${ }^{16}$

In today's crowded health and well-being marketplace, mindfulness has fared well by differentiating itself from other techniques that have occupied similar terrains. It has expanded its scientific credibility and increased its therapeutic applications, while selectively embracing its deeper Buddhist ethical framework to become embedded in a variety of clinical and nonclinical settings. Nonetheless, echoes of twentieth-century therapeutic relaxation strategies ring through recent mindfulness implementations. A recently launched mindfulness-based childbirth and parenting antenatal course, at the influential Oxford Mindfulness Centre, for example, includes 'learning how to work with pain and fear during childbirth', and 'managing stress... and other emotions, as a parent, and in everyday life'.

Recognizing the structural overlaps between relaxation and mindfulness interventions - notwithstanding their technical differences - offers important insights into why 'mind-body' therapeutic solutions to personal and social challenges are widely desired yet ambivalently received. Relaxation history elucidates the underlying socio-political conditions, and the popular and professional health and well-being ideologies, which have enabled and driven such practices to take root in our perpetually restless times. 
Acknowledgements This work was supported by the Wellcome Trust $[104411 / \mathrm{Z} / 14 / \mathrm{Z}]$.

\section{Notes}

1. 'Mindful Nation UK: Report by the Mindfulness All-Party Parliamentary Group (MAPPG)' (Mindfulness Initiative, October 2015), http://themindfulnessinitiative.org.uk/images/reports/Mindfulness-APPG-Report_ Mindful-Nation-UK_Oct2015.pdf.

2. Compare, for example, Kabat-Zinn's widely circulated audiovisual meditation The World of Relaxation: A Guided Mindfulness Meditation Practice for Healing in the Hospital and/or at Home (John Kabat-Zinn, 1982), with later declarations such as: '[Mindfulness] meditation is not relaxation spelled differently. Perhaps I should say that again... Meditation is not relaxation spelled differently' (Jon Kabat-Zinn, Coming to Our Senses: Healing Ourselves and the World through Mindfulness [New York, N.Y.: Hyperion, 2005], 58).

3. Edmund Jacobson, You Must Relax: A Practical Method of Reducing the Strains of Modern Living (New York ,N.Y.: McGraw-Hill, 1934), 8.

4. See E.J. Boome and M. A. Richardson, Relaxation in Everyday Life (London: Methuen \& Co. Ltd., 1938).

5. Ibid., 99.

6. Ornella Moscucci, 'Holistic Obstetrics: The Origins of "Natural Childbirth" in Britain', Postgraduate Medical Journal 79, no. 929 (2003): 168-73.

7. Original image available at Wellcome Images: https://wellcomeimages.org (search under L0079526). Image has not been modified in any way.

L0079526 Credit NCT/Wellcome Library, London, Wellcome Images Havering Branch: antenatal class photograph.

The National Childbirth Trust.

$\mathrm{SA} / \mathrm{NCT} / \mathrm{B} / 1 / 2 / 1 / 3 / 7$.

Photograph

From: National Childbirth Trust (NCT) 1952-2014

Collection: Archives \& Manuscripts

Library reference no.: Archives and Manuscripts SA/NCT/B/1/2/1/3/7 Copyrighted work available under CC BY 4.0

8. See Ayesha Nathoo, 'Initiating Therapeutic Relaxation in Britain: A Twentieth-Century Strategy for Health and Wellbeing', Palgrave Communications 2 (2016): 16,043, doi:10.1057/palcomms.2016.43 for a deeper discussion of the gendered uptake and application of relaxation therapies; and Meyer Friedman and Ray H. Rosenman, Type A Behavior and Your Heart (New York, N.Y.: Alfred Knopf, 1974) for more on 'Type A' behaviour and cardiac disease. 
9. Galen Ives, 'Relax', Journal of the Royal College of General Practitioners 28, no. 188 (1978): 187.

10. Karin Roon, The New Way to Relax (Kingswood: World's Work, 1951), 252.

11. Mark Singleton, 'Salvation through Relaxation: Proprioceptive Therapy and Its Relationship to Yoga', Journal of Contemporary Religion 20, no. 3 (2005): 289-304.

12. Geoffrey Harding, Relaxation and Healing, n.d., 9.

13. Herbert Benson, The Relaxation Response (N.Y.: Morrow, 1975).

14. On the transformation and appropriation of Buddhism and Buddhist meditation in the West, see David MacMahan, The Making of Buddhist Modernism (Oxford: Oxford University Press, 2008) and Jeff Wilson, Mindful America: The Mutual Transformation of Buddhist Meditation and American Culture (Oxford: Oxford University Press, 2014).

15. Jon Kabat-Zinn, 'Some Reflections on the Origins of MBSR, Skillful Means, and the Trouble with Maps', Contemporary Buddhism 12, no. 1 (2011): 282.

16. Ibid., 286-9.

Ayesha Nathoo is a medical historian at the Centre for Medical History, University of Exeter. Her current research examines the growth of therapeutic relaxation in twentieth-century Britain, in relation to chronic-disease prevention, pain management, and health and well-being advocacy.

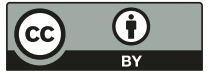

This chapter is distributed under the terms of the Creative Commons Attribution 4.0 International License (http://creativecommons.org/ licenses/by/4.0/), which permits use, duplication, adaptation, distribution and reproduction in any medium or format, as long as you give appropriate credit to the original author(s) and the source, a link is provided to the Creative Commons license and any changes made are indicated.

The images or other third party material in this chapter are included in the work's Creative Commons license, unless indicated otherwise in the credit line; if such material is not included in the work's Creative Commons license and the respective action is not permitted by statutory regulation, users will need to obtain permission from the license holder to duplicate, adapt or reproduce the material. 